\title{
Synuclein- $\gamma$ (SNCG) expression in ovarian cancer is associated with high-risk clinicopathologic disease
}

\author{
Anna Strohl ${ }^{1 \dagger}$, Kristina Mori ${ }^{1 \dagger}$, Stacey Akers ${ }^{2}$, Wiam Bshara ${ }^{3}$, Barbara Buttin ${ }^{4}$, Peter J. Frederick ${ }^{2}$, \\ Irene B. Helenowski ${ }^{5}$, Carl D Morrison ${ }^{3}$, Kunle Odunsi ${ }^{2}$, Julian C. Schink ${ }^{1}$, Denise M. Scholtens ${ }^{5}$, Jian-Jun Wei ${ }^{6,7^{*}}$ \\ and J. Julie $\mathrm{Kim}^{7 *}$
}

\begin{abstract}
Background: Synuclein gamma (SNCG) expression is associated with advanced disease and chemoresistance in multiple solid tumors. Our goal was to determine if SNCG protein expression in ovarian cancer was correlated with clinicopathologic variables and patient outcomes.

Methods: Tissue microarrays from primary tumors of 357 ovarian, fallopian tube, and primary peritoneal cancer patients, who underwent primary surgery at Roswell Park Cancer Institute between 1995 and 2007, were immunohistochemically stained for SNCG. A pathologist blinded to patient data scored tumors as positive if $\geq 10 \%$ of the sample stained for SNCG. Medical records were reviewed for clinicopathologic and demographic variables. Between the positive and negative groups, Wilcoxon rank-sum test was used to compare the median ages and Fisher's exact test was used to compare groups in categorical variables. Cox proportional hazard models examined associations between SNCG and overall and progression-free survival.

Results: The median follow-up was 36 months, median overall survival was 39 months, and median progression-free survival was 18 months. SNCG presence was associated with clinical variables of serous histology, grade 3 disease, suboptimal debulking, ascites at surgery, FIGO stage III-IV cancer, or initial CA-125 level >485. There was no significant difference in overall survival (HR $1.0695 \%$ Cl 0.81-1.39 P 0.69) or progression-free survival (HR $1.1695 \%$ Cl 0.89-1.50 $P$ 0.28) for patients with or without SNCG expression.
\end{abstract}

Conclusions: SNCG expression in ovarian cancer is frequent in patients with high-risk features, but it does not correlate with chemotherapy response, overall survival, or progression-free survival.

Keywords: Ovarian cancer, Synuclein gamma, High risk disease

\footnotetext{
*Correspondence: jianjun-wei@northwestern.edu; j-kim4@northwestern.edu

†'Equal contributors

${ }^{6}$ Department of Pathology, Robert H. Lurie Comprehensive Cancer Center,

Feinberg School of Medicine, Northwestern University, 251 East Huron Street, Feinberg 7-334, Chicago, IL 60611, USA

${ }^{7}$ Division of Reproductive Science in Medicine, Department of Obstetrics and

Gynecology, Robert H. Lurie Comprehensive Cancer Center, Northwestern

University Feinberg School of Medicine, 303 E. Superior Street, 4-117,

Chicago, IL 60611, USA

Full list of author information is available at the end of the article
} 


\section{Background}

Ovarian cancer is a leading cause of cancer mortality in women, accounting for more deaths than any other gynecologic malignancy in the United States [1]. While overall cancer incidence and mortality rates for gynecologic malignancies have declined in the past decade, progress in ovarian cancer outcomes has been slow. Despite developments in cytotoxic chemotherapy, five-year survival rates for women diagnosed with ovarian cancer remain less than $50 \%$ [2].

High rates of recurrence and associated mortality, coupled with advancements in the characterization of intracellular signaling pathways in carcinogenesis, have prompted investigation into other potential targets that can be used in the treatment of ovarian cancer, such as intracellular signaling pathways [3]. Among such targets, synuclein gamma (SNCG) was proposed as a potential target in ovarian cancer therapy [4].

Synucleins are a family of neuronal proteins expressed primarily in the peripheral nervous system. To date, three synuclein proteins have been identified: synuclein- $\alpha$ (SNCA), synuclein- $\beta$ (SNCB), synuclein- $\gamma$ (SNCG) $[5,6]$. The former two, SNCA and SNCB, have been implicated in neurodegenerative disorders, such as Parkinson's and Alzheimer's disease [7, 8], while SNCG has been primarily linked with cancer. SNCG was first discovered in breast cancer tissue [9] and has since been detected in multiple solid tumors, including breast, lung, liver, esophagus, colon, bladder, pancreatic, and prostate cancers [10]. SNCG has also been identified in gynecologic processes, including benign pathology (endometriosis), as well as endometrial and ovarian cancers $[10,11]$. SNCG expression occurs with advanced disease and chemoresistance in many cancers, and in breast cancer, SNCG has been causatively linked to increased proliferation, metastasis, and drug resistance [12].

Recent studies have demonstrated the role of synucleins as potential biomarkers in several cancer types, including ovarian cancer [13]. Several studies by immunohistochemistry (IHC) analysis demonstrated high levels of SNCG expression in up to $73 \%$ of epithelial ovarian cancers [1416] and one study showed that SNCG overexpression may promote the metastatic potential of ovarian cancer cells [17]. These findings suggest that SNCG may be a potential prognostic marker as well as a target for therapeutic drug development. Studies examining the correlation of SNCG expression with clinical outcomes are lacking, however, limited only to a single meta-analysis of gene expression profiles in ovarian cancer [18].

In this study, SNCG expression levels were examined using immunohistochemistry in primary and metastatic tumors of 357 patients with ovarian, fallopian tube, and primary peritoneal cancer. In particular, we examined the association of SNCG expression in ovarian cancer with worse outcomes, such as decreased progression free- and overall-survival and/or increased chemotherapy resistance.

\section{Methods \\ Patient population}

After obtaining IRB approval, the Pathology archive at Roswell Park Cancer Institute, Buffalo, New York was searched for ovarian, fallopian tube, and primary peritoneal cancer cases from 1995 to 2007. A chart review was conducted with extraction of clinical information, including patient's age at the time of diagnosis, the surgical stage, postoperative treatment. All patients underwent a primary surgical staging surgery, including total abdominal hysterectomy with bilateral salpingo-oophorectomy, with or without pelvic and para-aortic lymph nodes dissection and pelvic washings, depending on tumor stage. Patients were treated according to National Comprehensive Cancer Network guidelines (https://www.nccn.org). Patient's general information and tumor features are summarized in Table 1.

\section{Histologic evaluation and high-density tissue microarray (TMA) preparation}

Tumor grade was assessed using the International Federation of Gynecology and Obstetrics (FIGO) system and tumor stage was assigned based on the 2014 FIGO surgical staging guidelines. All slides were examined by a gynecologic pathologist for confirmation of tumor morphology and tumor grade. The viable tumor tissues and control tissues (fallopian tube) from each case were circled by pathologists. $0.6 \mathrm{~mm}$ tissue cores were punched and arrayed into high-density TMA receipting blocks.

\section{Immunohistochemistry}

Tissues were prepared for analysis, as previously described by Mhawech-Fauceglia et al. [19]. Briefly, high-density TMA blocks were sectioned in $4 \mu \mathrm{m}$ thickness followed by deparaffinization with xylene, then washed with ethanol. Sections were cooled for $20 \mathrm{~min}$ and incubated for 10 min with $3 \% \mathrm{H} 2 \mathrm{O} 2$ to quench endogenous peroxidase activity. Blocking was performed using serum-free protein block, Dakocytomation (Carpenteria, CA) for $30 \mathrm{~min}$. Antigen retrieval was done using a citrate-based buffer (pH 6; Bond Epitope Retrieval Solution, Leica Biosystems) and sections were incubated with the SNCG antibody (Abcam) for $1 \mathrm{~h}$ at room temperature on the Dako Autostainer Plus. The diaminobenzidine complex was used as a chromogen. Immunostained slides were blindly reviewed and scored by two gynecologic pathologists. Immunostain for SNCG was present in both the nucleus and cytoplasm. Immunoreactivity was semiquantitatively scored in immunointensity of 0 (negative), $1+$ (weak), $2+$ (moderate) and $3+$ (strong) and immunopercentage of $<10 \%, 10-50 \%$ and $>50 \%$. For the sake of statistical analysis, tumors were 
Table 1 Clinical and Pathologic Features of Patients

\begin{tabular}{ll}
\hline Variable & $N(\%)$ \\
\hline No. of patients & 357 \\
Follow up time, months & \\
Median & 36 \\
Range & $0-179$ \\
Age, year & \\
Median & 63 \\
Range & $22-93$ \\
CA125 level & \\
$\quad \leq 485$ & $141(53.2)$ \\
$>485$ & $124(46.8)$ \\
Ascites & \\
No & $73(29.1)$ \\
Present & $178(70.9)$ \\
Optimal Debulking Surgery & \\
Optimal & $245(82.5)$ \\
Suboptimal & $52(17.5)$ \\
Stage & \\
I & $33(9.8)$ \\
II & $31(9.2)$ \\
IV & $234(69.2)$ \\
Pil & $40(11.8)$
\end{tabular}

Primary Site of Disease

Ovarian

Primary Peritoneal

Fallopian Tube

Histologic subtype

Serous

Clear cell

Mucinous

Endometrioid

Other

Grade (FIGO)

2

3

Recurrence

No

Yes

SNCG Immunoexpression

Negative

$257(72)$

Positive

Status

Alive, No evidence of disease (ANED)

Alive, with evidence of disease (AWED)

$300(84.5)$

$53(14.9)$

$2(0.6)$

$262(73.6)$

$22(6.2)$

$12(3.4)$

$25(7.0)$

$35(9.8)$

7 (2.6)

$16(6.1)$

$240(91.3)$

$64(32.8)$

$131(67.2)$
Table 1 Clinical and Pathologic Features of Patients (Continued)

\begin{tabular}{ll}
\hline Died of Disease (DOD) & 268 (75.0) \\
Survival Time (months) & 39 \\
Median & $34-44$ \\
$95 \% \mathrm{Cl}$ & \\
Progression-free survival (months) & 18 \\
Median & $15-23$ \\
$95 \% \mathrm{Cl}$ & \\
\hline
\end{tabular}

Some clinical information were missing from cases and thus the sum of cases for each feature listed may not equate to 357

grouped as positive (SNCG+) or negative (SNCG-). Tumors were considered SNCG+ if $\geq 10 \%$ of the tumor epithelial cells were immunoreactive with immunointensity of $\geq 2$. Examples of positive and negative cases are illustrated in Fig. 1a-d.

Real time PCR

RNA from benign fallopian tube epithelium and ovarian cancer tissues was reverse transcribed. First-strand cDNA synthesis was performed using 700 ng of RNA and M-MLV reverse transcriptase (Life Technologies). Real time PCR was performed using Taqman reagent in a QuantStudio 5 system and primers to SNCG and the housekeeping gene TBP (Applied Biosystems/Life Technologies). Fold-change values were calculated using TBP as the housekeeping gene.

\section{Statistical analyses}

To test the association between the biomarker and the clinical parameters, Wilcoxon rank-sum test was used to compare the median ages and Fisher's exact test was used to compare frequencies and percentages for categorical variables. Progression-free survival (PFS), was defined as observed length of time from date of diagnosis to recurrence or censoring at date of last contact. Overall survival (OS) was defined as observed length of life from date of diagnosis to death or censoring at date of last contact. For survival analysis, Kaplan-Meier curves were used to estimate PFS, and OS curves. Curves were compared for tumors with and without SNCG expression using the logrank test. Univariate cox proportional hazards models were used to estimate hazard ratios for SNCG status for both the OS and PFS outcomes. Multivariate Cox regression was then conducted with SNCG expression as the main predictor and adjustment for clinical factors that were associated with SNCG expression (CA125 level, ascites, debulking status, FIGO grade, tumor stage and histologic subtype). Since several of the clinical covariates were highly associated with each other, sensitivity of the hazard ratio es67 (18.8) timates for SNCG was examined after adjustment for each 22 (6.2) clinical variable in separate models. All reported $p$ values are two sided with $P<0.05$ significance. Statistical analyses 

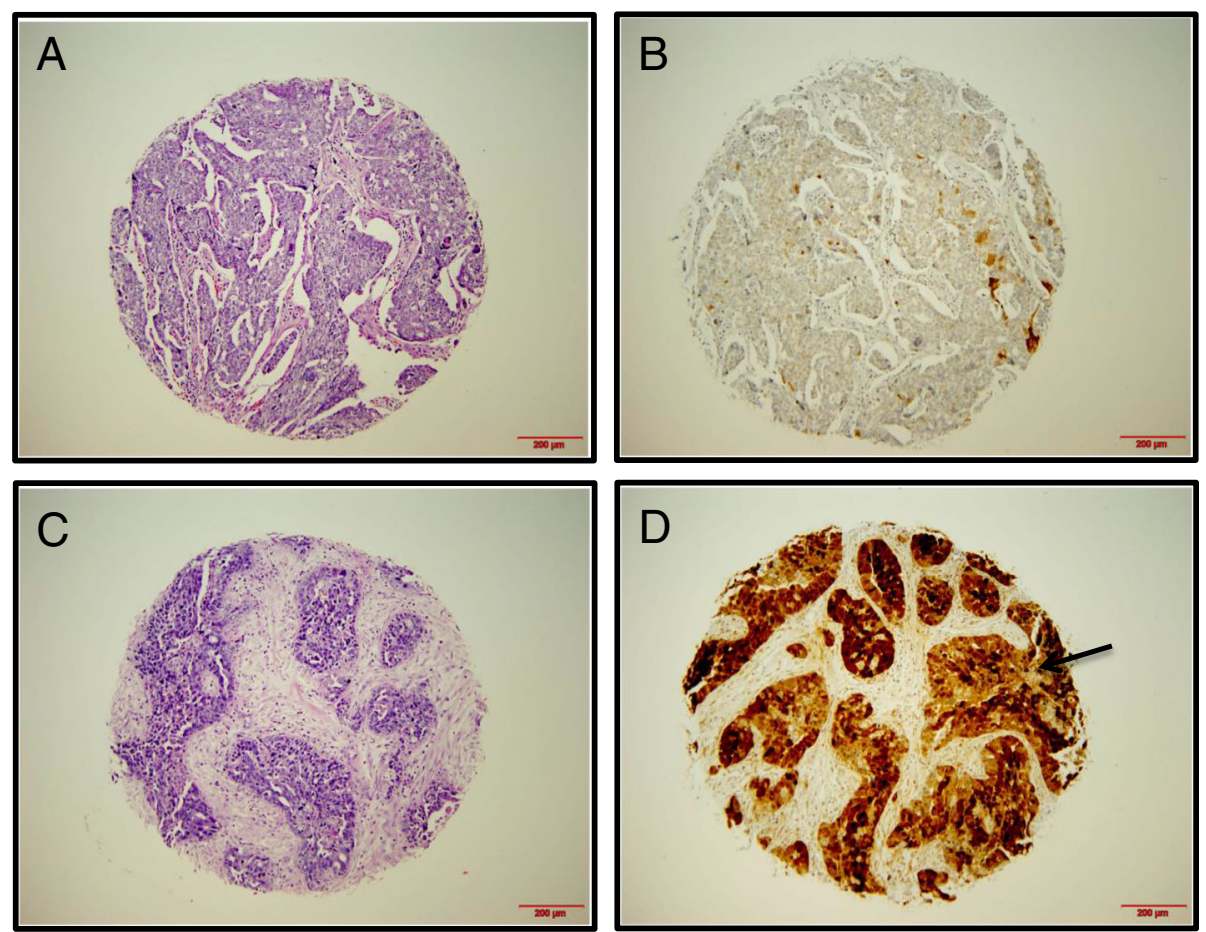

Fig. 1 Immunohistochemical staining of SNCG in ovarian tumor sections in the TMA. Representative sections of negative $\mathbf{b}$ and positive $\mathbf{d}$ SNCG expression with corresponding H\&E staining a, c are shown. Brown color (arrow) represents positive staining for SNCG

was performed using SAS v 9.4. Unpaired $t$-test was used to compare SNCG mRNA levels in benign fallopian tube and ovarian cancer tissues using Graphpad Prism version 6.0 (Graphpad Software, La Jolla, CA, USA).

\section{Results}

\section{Patient characteristics}

The clinical and pathologic features of 357 patients with primary ovarian, fallopian tube, and primary peritoneal cancer are summarized in Table 1 . The mean age at diagnosis was 63 years. All patients $(n=357)$ underwent primary surgery with $82.5 \%$ optimal debulking. No patients received neoadjuvant chemotherapy or radiation therapy. The majority of patients had advanced stage (FIGO Stage III/IV: $81.1 \%$ ), were serous type (73.6 \%) and high-grade (FIGO Grade 3: $91.3 \%$ ) disease. About $70 \%$ of patients had preoperative ascites and $46.8 \%$ had CA-125 levels $>485 \mathrm{U} / \mathrm{mL}$ at the time of surgery. Median follow-up time was 36 months (range 0-179 months). At the time of data analysis, $67.2 \%$ of patients had experienced disease recurrence and $24.9 \%$ were surviving.

\section{Clinical characteristics and SNCG expression}

SNCG expression was positive in $72 \%$ (257/357) of primary tumors. Examples of positive and negative cases are illustrated in Fig. 1a-d. SNCG was expressed in different types of ovarian cancers as well, including fallopian tube, endometrioid carcinoma, clear cell carcinoma, and low grade serous carcinoma (Fig. 2). SNCG overexpression was associated with tumor type, stage, grade and other clinical parameters (Table 2). SNCG overexpression was significantly higher in cases with serous histology than in other histologic variants $(p<0.0001)$. In 212 cases with serous histology, $85 \%$ of cases were SNCG positive while only $43 \%$ of other types were SNCG positive. Similarly, SNCG overexpression was much more common in high grade tumors $(73 \%)$ than in low grade $(47 \%)(p=0.01)$. Stage III/IV disease had significantly high SNCG expression in comparison to stage I/II $(p<0.0001)$. About $88 \%$ of suboptimal debulking tumors showed SNCG overexpression in comparison to $69 \%$ of optimal debulking cases $(p=0.004)$. Patients with ascites also showed significantly higher SNCG expression than those without ascites $(p=0.01)$. When dividing CA-125 scores of below and above 485, SNCG expression was marginally associated with high CA-125 expression as well $(p=0.02)$. There was no association between age at diagnosis or site of primary disease and SNCG expression.

To provide a quantitative measure of SNCG levels using real time PCR, RNA from tissue samples outside of the cases used for the tissue microarray, was analyzed. SNCG expression from benign human fallopian tube epithelium and ovarian cancer tissues was compared. Benign fallopian tube epithelium expressed less SNCG 


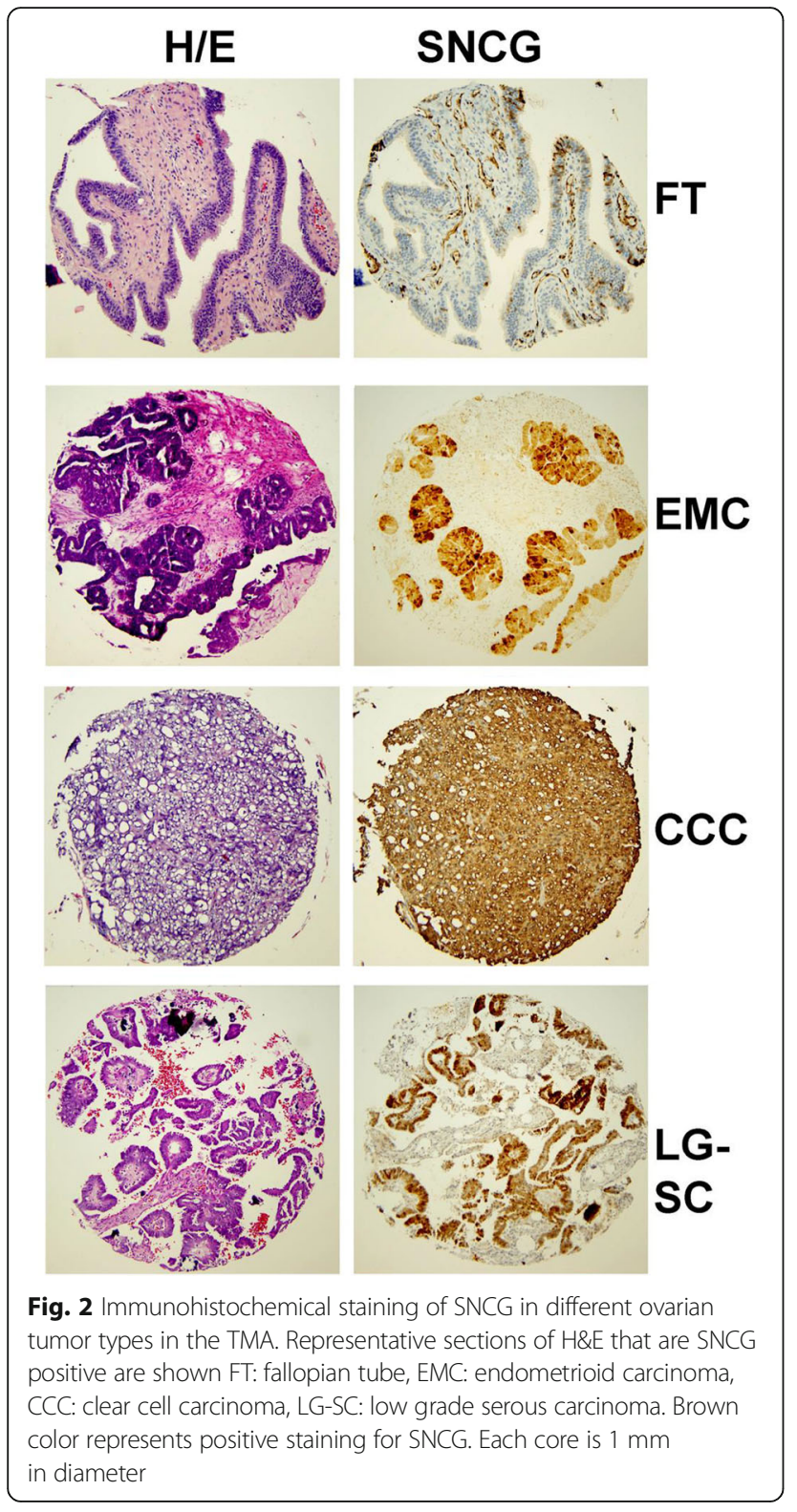

than ovarian cancer tissues (Additional file 1: Figure S1), which supports the immunohistochemical analysis of SNCG (Fig. 2).

\section{Survival analyses}

The median OS for the entire study group was 39 months (95 \% CI: 34-44 months) and 5-years OS was $31.57 \%$. Median progression-free survival (PFS) was 18 months (95 \% CI: 15-23). There was no significant difference in OS (HR 1.06; $95 \%$ CI $0.81-1.39, p=0.69$ ) or PFS (HR 1.16; $95 \%$ CI $0.89-1.50, p=0.28$ ) for patients with SNCG+ compared to those with SNCG- tumors (Fig. 3). The lack of association between SNCG expression and OS and PFS persisted after adjustment for all clinical variables associated with SNCG (Additional file 2: Table S1).
Table 2 Clinical Factors by SNCG expression status

\begin{tabular}{|c|c|c|c|}
\hline & SNCG positive & SNCG negative & $p$-value \\
\hline No. of patients $(n=357)$ & $257(72 \%)$ & $100(28 \%)$ & \\
\hline \multicolumn{4}{|l|}{ Age, year } \\
\hline Median & 63.0 & 63.5 & \multirow[t]{2}{*}{0.93} \\
\hline Range & $22-91$ & $36-93$ & \\
\hline \multicolumn{4}{|l|}{ CA125 level } \\
\hline$\leq 485$ & $93(48.7 \%)$ & 48 (64.9\%) & \multirow[t]{2}{*}{0.02} \\
\hline$>485$ & $98(51.3 \%)$ & 26 (35.1\%) & \\
\hline \multicolumn{4}{|l|}{ Ascites } \\
\hline No & 44 (24.4\%) & $29(40.8 \%)$ & \multirow[t]{2}{*}{0.01} \\
\hline Present & $136(75.6 \%)$ & $42(59.2 \%)$ & \\
\hline \multicolumn{4}{|l|}{ Debulking Status } \\
\hline Optimal & $169(78.6 \%)$ & 76 (92.7 \%) & \multirow[t]{2}{*}{0.004} \\
\hline Suboptimal & $46(21.4 \%)$ & $6(7.3 \%)$ & \\
\hline \multicolumn{4}{|l|}{ Stage } \\
\hline$|/| \mid$ & 30 (12.2 \%) & 34 (36.6 \%) & \multirow[t]{2}{*}{$<0.0001$} \\
\hline III/IV & $215(87.8 \%)$ & $59(63.4 \%)$ & \\
\hline \multicolumn{4}{|l|}{ Primary Site of Disease } \\
\hline Ovarian & $219(85.9 \%)$ & $81(81.0 \%)$ & \multirow[t]{3}{*}{0.28} \\
\hline Primary Peritoneal & $34(13.3 \%)$ & 19 (19.0\%) & \\
\hline Fallopian Tube & $2(0.8 \%)$ & $0(0.0 \%)$ & \\
\hline \multicolumn{4}{|l|}{ Histologic subtype } \\
\hline Serous & $181(82.7 \%)$ & $31(38.3 \%)$ & \multirow[t]{2}{*}{$<0.0001$} \\
\hline Non-serous & $38(17.3 \%)$ & $50(61.7 \%)$ & \\
\hline \multicolumn{4}{|l|}{ Grade (FIGO) } \\
\hline $1 / 2$ & $11(5.8 \%)$ & $12(16.0 \%)$ & \multirow[t]{2}{*}{0.01} \\
\hline 3 & 177 (94.2\%) & $63(84.0 \%)$ & \\
\hline \multicolumn{4}{|l|}{ Recurrence } \\
\hline No & $23(19.49 \%)$ & $15(29.41 \%)$ & \multirow[t]{2}{*}{0.17} \\
\hline Yes & 95 (80.51 \%) & $36(70.59 \%)$ & \\
\hline \multicolumn{4}{|l|}{ Status } \\
\hline Alive & 60 (23.3 \%) & 29 (29.0\%) & \multirow[t]{2}{*}{0.28} \\
\hline Dead & 197 (76.7 \%) & 71 (71.0 \%) & \\
\hline
\end{tabular}

Percentages are based on denominators of available data due to missing information from cases

\section{Metastatic disease}

We further explored SNCG expression in metastases. Among patients with metastatic tumor samples $(n=184)$, $153(83 \%)$ of metastatic tumors demonstrated SNCG expression. The presence of SNCG expression was compared between primary and metastatic tumors within the same patient: $12(6.6 \%)$ metastatic tumors gained expression of SNCG, 34 (18.6 \%) lost expression, 119 (64 \%) maintained expression, and 19 (10.4\%) never demonstrated SNCG expression (Table 3). The 5 -years OS by groups were: $41.67 \%$ for tumors gained expression of SNCG, $32.09 \%$ for lost expression, $20.59 \%$ for maintained expression, and $21.05 \%$ 

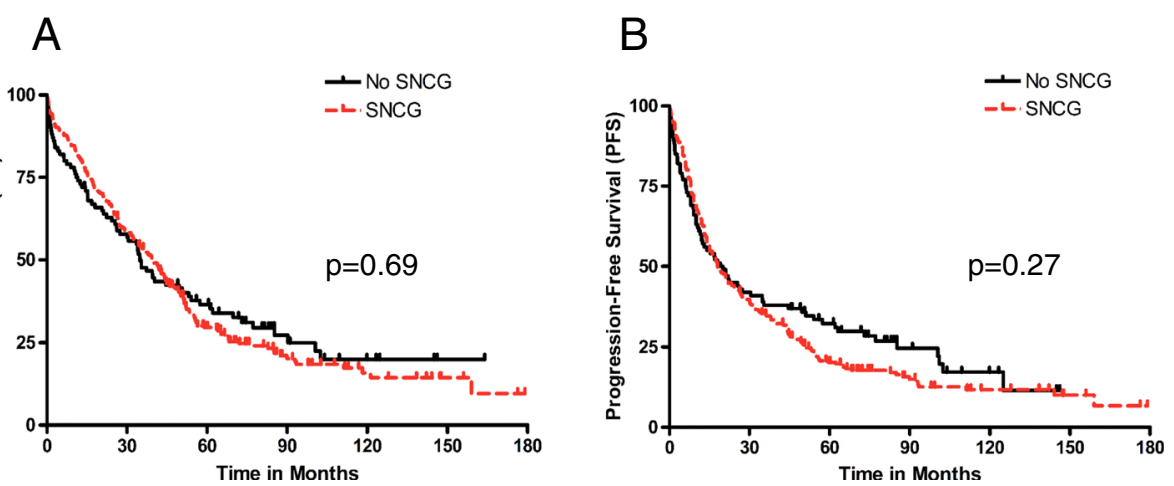

Fig. 3 Kaplan-Meier survival curves for patients with ovarian cancer stratified according to SNCG expression (positive versus negative). Curves for $\mathbf{a}$ overall survival and $\mathbf{b}$ progression-free survival are shown

never demonstrated SNCG expression. There was no significant difference in OS among patients in whom primary tumors gained SNCG expression, lost SNCG expression, maintained SNCG expression, or never expressed SNCG $(\log -\operatorname{rank} p=0.23)$.

\section{SNCG expression and chemotherapy response/platinum resistance}

We further explored the impact of SNCG on chemotherapy response. Table 4 demonstrates the distribution of SNCG expression by platinum-refractory (defined as disease progression with first-line chemotherapy) or platinum-sensitive (defined as disease recurrence within 6 months of first-line chemotherapy) disease status compared to those patients with no evidence of disease (NED). Results show a marginally greater prevalence of platinum-refractory cases in cases with positive SNCG expression $(p=0.08)$. There was no significant difference in SNCG status in patients with platinum-sensitive disease $(p=0.79)$.

\section{Discussion}

This study is the first to formally evaluate the proposed association between SNCG protein expression and clinical outcomes in patients with ovarian, fallopian tube, and primary peritoneal cancers. SNCG is a new potential biomarker and demonstrates to be specific and reliable for immunohistochemistry in formalin-fixed and paraffinembedded tissue sections. SNCG has been examined in several different tumor types [10, 13-16]. Although the cut-off value of immunoreactivity for positive and negative SNCG has not been established for each tumor type, we found it was meaningful and reproducible in ovarian cancer when $10 \%$ of tumor cells have moderate to strong immunoreactivity for SNCG. In our series of 357 patients, expression of SNCG was identified in $72 \%$ of primary tumors, a percentage similar to the $73 \%$ expression that has previously been reported in ovarian carcinomas [14]. The hypothesis, namely that SNCG expression was associated with worse clinical outcomes, was refuted, as the results of our study found no association between SNCG expression and OS or PFS in primary tumors.

While SNCG expression did not correlate with clinical outcomes such as PFS, OS, or chemoresistance, the results of our study did find that there was a significant association between SNCG expression and high-risk clinicopathological factors, such as serous histology, high grade disease, advanced stage, and suboptimal debulking surgery. These associations suggest that while SNCG expression might not be a single prognostic marker, it

Table 3 SNCG Expression in Metastases of Primary Tumors

\begin{tabular}{|c|c|c|c|c|c|}
\hline Primary/Metastasis & $\mathrm{N}$ & $\mathrm{N}$ event & Median OS (months) & 2 years OS & 5 years OS \\
\hline$-/+$ (Gained expression) & 12 & 10 & $47(30,102)$ & $91.67 \%$ & $41.67 \%$ \\
\hline +/- (Lost expression) & 34 & 26 & $32(17,55)$ & $58.82 \%$ & $32.09 \%$ \\
\hline +/+ (Maintained expression) & 118 & 104 & $39(27,44)$ & $65.25 \%$ & $20.59 \%$ \\
\hline$-/-($ Never expressed) & 19 & 17 & $17(9,44)$ & $47.37 \%$ & $21.05 \%$ \\
\hline \multicolumn{6}{|l|}{ Log-rank $p=0.23$} \\
\hline \multicolumn{6}{|c|}{ Combined Metastatic Expression } \\
\hline Positive & 130 & 114 & $40(29,44)$ & $67.69 \%$ & $22.65 \%$ \\
\hline Negative & 53 & 43 & $29(15,44)$ & $54.72 \%$ & $28.18 \%$ \\
\hline
\end{tabular}


Table 4 SNCG expression in response to chemotherapy. Comparisons between patients with platinum-refractory (defined as disease progression with first-line chemotherapy) and patients with no evidence of disease (NED) or platinum-sensitive disease status (defined as disease recurrence within 6 months of first-line chemotherapy) and platinum-resistant are shown

\begin{tabular}{clll}
\hline SNCG Expression & $\begin{array}{l}\text { Platinum-refractory } \\
(N=107)\end{array}$ & $\begin{array}{l}\text { NED } \\
(N=164)\end{array}$ & $p$-value \\
No & $20(18.7 \%)$ & $46(28.0 \%)$ & 0.08 \\
Yes & $87(81.3 \%)$ & $118(72.0 \%)$ & \\
SNCG Expression & $\begin{array}{l}\text { Platinum sensitive } \\
(N=145)\end{array}$ & $\begin{array}{l}\text { Platinum resistant } \\
(N=19)\end{array}$ & \\
No & $40(27.6 \%)$ & $6(31.6 \%)$ & 0.79 \\
Yes & $105(72.4 \%)$ & $13(68.4 \%)$ & \\
\hline
\end{tabular}

may be an indicator for high-risk disease and may play a role in the pathogenesis of disease progression. Furthermore, SNCG expression may provide additional evidence of disease burden, given its association with advanced high-grade disease. Given its potential as a marker for high-risk disease, future studies should focus on the potential role of secretory SNCG a surrogate marker for disease burden, such as CA125. Prior studies have demonstrated that SNCG is detectable in the serum of patients with cancer [20-23]. The next direction would be to test serum samples from patients that were collected at the same time as tumor tissue in order to correlate serum and tissue SNCG levels.

In regards to SNCG expression in metastatic ovarian cancer, our data did not demonstrate a significant difference in OS among patients in whom primary tumors gained or lost SNCG expression; however, our data did demonstrate that the majority of metastatic tumors (83 \%) demonstrated SNCG expression. This data supports prior studies that implicate SNCG overexpression may promote the metastatic potential of ovarian cancer cells [17] and further supports the association of SNCG expression with high-risk clinicopathologic disease. Our findings of high levels of SNCG expression in ovarian cancer were consistent with SNCG expression in multiple cancer types, including breast, liver, prostate, and colon cancer [14, 21, 24, 25]. However, unlike breast [12] and endometrial cancers [19] in which high SNCG expression has been correlated with adverse clinical outcomes, our study found no correlation between high SNCG expression and clinical outcomes.

There are several possibilities for the lack of association with OS or PFS in this study in ovarian cancer. First, the study may have been underpowered to detect a significant difference in clinical outcomes. The association of SNCG expression with high-risk disease would suggest that there is a difference in clinical outcomes such as PFS or OS, however this was not seen in our cohort. Next, ovarian cancer is a heterogeneous cancer with multiple histologic subtypes and underlying molecular aberrations [26] and thus SNCG expression may influence tumor phenotype differently depending on co-existing histologic and molecular factors. Furthermore, as SNCG expression is not specific to ovarian cancer, its function in ovarian cancer may be a feature of malignancy in general. Finally, we evaluated the presence of SNCG, not its function, nor its level of expression. Tumors may change such genomic outcomes and our study is insufficient to determine either the underlying somatic or germline polymorphisms or the epigenetic actions on SNCG genes. Future studies might focus on the correlation of SNCG protein and gene expression in combination with other markers of disease, such as CA125 levels.

In conclusion, this study found that while SNCG expression is often present in ovarian carcinoma, the positive or negative expression of SNCG protein alone is not independently associated with clinical prognosis. Our results did indicate that SNCG expression is associated with clinicopathologic features of high-risk disease, suggesting that SNCG expression may play a role in severity of disease and be a marker for aggressive disease. Furthermore, as targeted drug therapy develops, recognition of SNGC expression in ovarian cancer will remain important as we continue to discover ways to improve outcomes in patients diagnosed with this deadly disease.

\section{Conclusions}

Expression of SNCG is associated with clinicopathologic variables of aggressive and advanced disease but not with overall survival or progression free survival. SNCG may serve as a novel biomarker for aggressive or advanced ovarian carcinoma and warrants further investigation to determine its role in this disease.

\section{Additional files}

Additional file 1: Figure S1. Expression of SNCG mRNA in benign fallopian tube epithelium and ovarian cancer tissues. RNA from human benign fallopian tube epithelium and ovarian cancer tissues was subjected to RT-real time PCR for SNCG. SNCG as a ratio of the housekeeping gene TBP is presented using delta delta CT calculations. ${ }^{*}=p<0.05$. FT - fallopian tube; OvCa - ovarian cancer. (PPTX 64 kb)

Additional file 2: Table S1. Hazard ratios (HR) from multivariate Cox regression models for PFS and OS involving SNCG expression and adjusting for relevant demographic and clinical factors. (DOCX $123 \mathrm{~kb}$ )

\section{Abbreviations}

CA-125: Cancer antigen-125; FIGO: International Federation of Gynecology and Obstetrics; HR: Hazard ratio; NED: No evidence of disease; OS: Overall survival; PFS: Progression-free survival; SNCA: Synuclein-alpha;

SNCB: Synuclein-beta; SNCG: Synuclein gamma; TMA: Tissue microarray; U/ $\mathrm{mL}$ : Units per milliliter; Yr: Year 


\section{Acknowledgements}

We would like to acknowledge the Pathology Core Facility at Northwestern University for assistance with tissue microarray and immunohistochemistry staining. We also are grateful to Ms. Zhenxiao Lu from the Kim lab for technical assistance.

\section{Funding}

This work was funded by the Rivkin Ovarian Cancer Fund (JJW).

\section{Availability of data and materials}

The datasets supporting the conclusions of this article are included within the article.

\section{Authors' contribution}

$A S, K M, J J K, B B, J J W, J C S$ were involved in the conception and design of the study. JJW, BB, WB, PJF, KO, CM, SA were involved in the acquisition of cases and tissue microarray construction. AS, KM, JJK, JJW, IBH, DMS analyzed and interpreted the data. AS, KM, JJK, BB, JJW, JCS, WB, PJF, KO, CM, SA, IBH, DMS were involved in the writing, review and revision of manuscript. Study was supervised by JJK and JJW. All authors read and approved the final manuscript.

\section{Competing interests}

The authors declare that they have no competing interests.

\section{Consent for publication}

Not applicable.

\section{Ethics approval and consent to participate}

This study was approved by the Institutional Review Board at Roswell Park Cancer Institute in accordance with U.S. Department of Health Regulations.

\section{Author details}

'Division of Gynecologic Oncology, Department of Obstetrics and Gynecology, Robert H. Lurie Comprehensive Cancer Center, Northwestern University Feinberg School of Medicine, Chicago, USA. ${ }^{2}$ Division of Gynecologic Oncology, Roswell Park Cancer Institute, Buffalo, USA. ${ }^{3}$ Department of Pathology, Roswell Park Cancer Institute, Buffalo, USA ${ }^{4}$ Gynecologic Oncology Program, Cadence Physician Group, Warrenville, USA. ${ }^{5}$ Department of Preventive Medicine, Northwestern University Feinberg School of Medicine, Chicago, USA. ${ }^{6}$ Department of Pathology, Robert H. Lurie Comprehensive Cancer Center, Feinberg School of Medicine, Northwestern University, 251 East Huron Street, Feinberg 7-334, Chicago, IL 60611, USA. ${ }^{7}$ Division of Reproductive Science in Medicine, Department of Obstetrics and Gynecology, Robert H. Lurie Comprehensive Cancer Center, Northwestern University Feinberg School of Medicine, 303 E. Superior Street, 4-117, Chicago, IL 60611, USA.

Received: 3 August 2016 Accepted: 17 October 2016

Published online: 03 November 2016

\section{References}

1. Jayson GC, Kohn EC, Kitchener HC, Ledermann JA. Ovarian cancer. Lancet. 2014;384(9951):1376-88.

2. Howlader NNA, Krapcho M, Miller D, Bishop K, Altekruse SF, Kosary CL, Yu M, Ruhl J, Tatalovich Z, Mariotto A, Lewis DR, Chen HS, Feuer EJ, Cronin KA. SEER Cancer Statistics Review, 1975-2013. In: National Cancer Institute. 2016.

3. Nicosia SV, Bai W, Cheng JQ, Coppola D, Kruk PA. Oncogenic pathways implicated in ovarian epithelial cancer. Hematol Oncol Clin North Am. 2003;17(4):927-43.

4. Singh VK, Jia Z. Targeting synuclein-gamma to counteract drug resistance in cancer. Expert Opin Ther Targets. 2008;12(1):59-68.

5. George JM. The synucleins. Genome Biol. 2002;3(1):REVIEWS3002.

6. Lavedan C. The synuclein family. Genome Res. 1998;8(9):871-80.

7. Iwai A. Properties of NACP/alpha-synuclein and its role in Alzheimer's disease. Biochim Biophys Acta. 2000;1502(1):95-109.

8. von Bohlen Und Halbach O. Synucleins and their relationship to Parkinson's disease. Cell Tissue Res. 2004:318(1):163-74.

9. Ji H, Liu YE, Jia T, Wang M, Liu J, Xiao G, Joseph BK, Rosen C, Shi YE. Identification of a breast cancer-specific gene, BCSG1, by direct differential cDNA sequencing. Cancer Res. 1997;57(4):759-64.
10. Liu H, Liu W, Wu Y, Zhou Y, Xue R, Luo C, Wang L, Zhao W, Jiang JD, Liu J. Loss of epigenetic control of synuclein-gamma gene as a molecular indicator of metastasis in a wide range of human cancers. Cancer Res. 2005;65(17):7635-43.

11. Singh MN, Stringfellow HF, Taylor SE, Ashton KM, Ahmad M, Abdo KR, ElAgnaf OM, Martin-Hirsch PL, Martin FL. Elevated expression of CYP1A1 and gamma-SYNUCLEIN in human ectopic (ovarian) endometriosis compared with eutopic endometrium. Mol Hum Reprod. 2008;14(1 1):655-63.

12. Jia T, Liu YE, Liu J, Shi YE. Stimulation of breast cancer invasion and metastasis by synuclein gamma. Cancer Res. 1999;59(3):742-7.

13. Ahmad M, Attoub S, Singh MN, Martin FL, El-Agnaf OM. Gamma-synuclein and the progression of cancer. FASEB J. 2007:21(13):3419-30.

14. Bruening W, Giasson BI, Klein-Szanto AJ, Lee VM, Trojanowski JQ, Godwin AK. Synucleins are expressed in the majority of breast and ovarian carcinomas and in preneoplastic lesions of the ovary. Cancer. 2000;88(9):2154-63.

15. Czekierdowski A, Czekierdowska S, Danilos J, Czuba B, Sodowski K Sodowska H, Szymanski M, Kotarski J. Microvessel density and CpG island methylation of the THBS2 gene in malignant ovarian tumors. J Physiol Pharmacol. 2008;59 Suppl 4:53-65.

16. Gupta A, Godwin AK, Vanderveer L, Lu A, Liu J. Hypomethylation of the synuclein gamma gene CpG island promotes its aberrant expression in breast carcinoma and ovarian carcinoma. Cancer Res. 2003;63(3):664-73.

17. Cheung KT, Taylor SE, Patel II, Bentley AJ, Stringfellow HF, Fullwood NJ, Martin-Hirsch PL, Martin FL. Expression of ERalpha, its ERalphaDelta3 Splice Variant and gamma-SYNUCLEIN in Ovarian Cancer: A Pilot Study. Br J Med Med Res. 2011;1(4):430-44.

18. Fekete T, Raso E, Pete I, Tegze B, Liko I, Munkacsy G, Sipos N, Rigo Jr t, Gyorffy B. Meta-analysis of gene expression profiles associated with histological classification and survival in 829 ovarian cancer samples. Int J Cancer. 2012;131(1):95-105.

19. Mhawech-Fauceglia P, Wang D, Syriac S, Godoy H, Dupont N, Liu S, Odunsi K. Synuclein-gamma (SNCG) protein expression is associated with poor outcome in endometrial adenocarcinoma. Gynecol Oncol. 2012;124(1):148-52.

20. Iwaki H, Kageyama S, Isono T, Wakabayashi Y, Okada Y, Yoshimura K, Terai A, Arai $\mathrm{Y}$, Iwamura $\mathrm{H}$, Kawakita M, et al. Diagnostic potential in bladder cancer of a panel of tumor markers (calreticulin, gamma -synuclein, and catechol-omethyltransferase) identified by proteomic analysis. Cancer Sci. 2004;95(12):955-61.

21. Li Z, Sclabas GM, Peng B, Hess KR, Abbruzzese JL, Evans DB, Chiao PJ. Overexpression of synuclein-gamma in pancreatic adenocarcinoma. Cancer. 2004;101(1):58-65

22. Liu C, Guo J, Qu L, Bing D, Meng L, Wu J, Shou C. Applications of novel monoclonal antibodies specific for synuclein-gamma in evaluating its levels in sera and cancer tissues from colorectal cancer patients. Cancer Lett. 2008; 269(1):148-58

23. Liu C, Qu L, Lian S, Tian Z, Zhao C, Meng L, Shou C. Unconventional secretion of synuclein-gamma promotes tumor cell invasion. FEBS J. 2014;281(22):5159-71.

24. Wu K, Weng Z, Tao Q, Lin G, Wu X, Qian H, Zhang Y, Ding X, Jiang Y, Shi YE. Stage-specific expression of breast cancer-specific gene gamma-synuclein. Cancer Epidemiol Biomarkers Prev. 2003;12(9):920-5.

25. Ye Q, Wang TF, Peng YF, Xie J, Feng B, Qiu MY, Li LH, Lu AG, Liu BY, Zheng $\mathrm{MH}$. Expression of alpha-, beta- and gamma-synuclein in colorectal cancer, and potential clinical significance in progression of the disease. Oncol Rep. 2010;23(2):429-36.

26. Cancer Genome Atlas Research N. Integrated genomic analyses of ovarian carcinoma. Nature. 2011;474(7353):609-15.

\section{Submit your next manuscript to BioMed Central and we will help you at every step:}

- We accept pre-submission inquiries

- Our selector tool helps you to find the most relevant journal

- We provide round the clock customer support

- Convenient online submission

- Thorough peer review

- Inclusion in PubMed and all major indexing services

- Maximum visibility for your research

Submit your manuscript at www.biomedcentral.com/submit
) Biomed Central 